\title{
On the number of non-isomorphic subspaces of a Banach space
}

\author{
by \\ VAlentin Ferenczi (Paris) and \\ Christian Rosendal (Paris and Pasadena, CA)
}

\begin{abstract}
We study the number of non-isomorphic subspaces of a given Banach space. Our main result is the following. Let $\mathfrak{X}$ be a Banach space with an unconditional basis $\left(e_{i}\right)_{i \in \mathbb{N}}$; then either there exists a perfect set $P$ of infinite subsets of $\mathbb{N}$ such that for any two distinct $A, B \in P,\left[e_{i}\right]_{i \in A} \not\left[e_{i}\right]_{i \in B}$, or for a residual set of infinite subsets $A$ of $\mathbb{N},\left[e_{i}\right]_{i \in A}$ is isomorphic to $\mathfrak{X}$, and in that case, $\mathfrak{X}$ is isomorphic to its square, to its hyperplanes, uniformly isomorphic to $\mathfrak{X} \oplus\left[e_{i}\right]_{i \in D}$ for any $D \subset \mathbb{N}$, and isomorphic to a denumerable Schauder decomposition into uniformly isomorphic copies of itself.
\end{abstract}

The starting point of this article is the so-called "homogeneous space problem", due to S. Banach. It was solved at the end of the last century by W. T. Gowers [3], [4], R. Komorowski and N. Tomczak-Jaegermann [8] and W. T. Gowers and B. Maurey [5]; see, e.g., [10] for a survey. Recall that a Banach space is said to be homogeneous if it is isomorphic to all of its (infinite-dimensional closed) subspaces. The previously named authors showed that $\ell_{2}$ is the only homogeneous Banach space. A very natural question was posed to us by G. Godefroy: if a Banach space is not isomorphic to $\ell_{2}$, then how many mutually non-isomorphic subspaces must it contain? (obviously, at least 2). In this article, we concentrate on spaces with a basis and subspaces of it spanned by subsequences. We shall also be interested in the relation of equivalence of basic sequences. By "many" we shall mean the Cantor concept of cardinality, and sometimes finer concepts from the theory of classification of equivalence relations in descriptive set theory.

1. Basic notions about basic sequences. Let $\mathfrak{X}$ be a separable Banach space and $\left(e_{i}\right)_{i \in \mathbb{N}}$ a sequence in $\mathfrak{X}$. We say that $\left(e_{i}\right)_{i \in \mathbb{N}}$ is a basis for $\mathfrak{X}$ if any vector $x$ in $\mathfrak{X}$ can be uniquely written as a norm convergent series $x=\sum_{i \in \mathbb{N}} a_{i} e_{i}$. In that case the functionals $e_{k}^{*}\left(\sum_{i \in \mathbb{N}} a_{i} e_{i}\right):=a_{k}$ are in fact continuous, as are the projections $P_{n}\left(\sum_{i \in \mathbb{N}} a_{i} e_{i}\right):=\sum_{i=0}^{n} a_{i} e_{i}$, and further-

2000 Mathematics Subject Classification: Primary 46B03; Secondary 03A15. 
more their norms are uniformly bounded, $\sup _{n}\left\|P_{n}\right\|<\infty$. The supremum is called the basis constant of $\left(e_{i}\right)_{i \in \mathbb{N}}$ and is denoted by bc $\left(\left(e_{i}\right)_{i \in \mathbb{N}}\right)$. If $\left(e_{i}\right)_{i \in \mathbb{N}}$ is a sequence that is a basis for its closed linear span, written $\left[e_{i}\right]_{i \in \mathbb{N}}$, we say that it is a basic sequence in $\mathfrak{X}$ and its basis constant is defined as before. The property of $\left(e_{i}\right)_{i \in \mathbb{N}}$ being a basic sequence can also be equivalently stated as the existence of a constant $K \geq 1$ such that for any $n \leq m$ and $a_{0}, a_{1}, \ldots, a_{m} \in \mathbb{R}$

$$
\left\|\sum_{i=0}^{n} a_{i} e_{i}\right\| \leq K\left\|\sum_{i=0}^{m} a_{i} e_{i}\right\| .
$$

The infimum of such $K$ will then be the basis constant bc $\left(\left(e_{i}\right)_{i \in \mathbb{N}}\right)$. Suppose furthermore that for any $x=\sum_{i \in \mathbb{N}} a_{i} e_{i}$ the series actually converges unconditionally, i.e., for any permutation $\sigma$ of $\mathbb{N}$ the series $\sum_{i \in \mathbb{N}} a_{\sigma(i)} e_{\sigma(i)}$ converges to $x$. Then the basic sequence is said to be unconditional. Again being an unconditional basis for some closed subspace (which will be designated by "unconditional basic sequence") is equivalent to there being a constant $K \geq 1$ such that for all $n, A \subset\{0, \ldots, n\}$ and $a_{0}, \ldots, a_{n} \in \mathbb{R}$,

$$
\left\|\sum_{i \in A} a_{i} e_{i}\right\| \leq K\left\|\sum_{i=0}^{n} a_{i} e_{i}\right\| .
$$

We will in general only work with normalised basic sequences, i.e., $\left\|e_{i}\right\| \equiv 1$, which can always be obtained by taking $e_{i}^{\prime}:=e_{i} /\left\|e_{i}\right\|$.

Two sequences $\left(e_{i}\right)$ and $\left(f_{i}\right)$ are said to be equivalent if there exist $C$ and $a, b$ with $a b \leq C$ such that $(1 / a)\left\|\sum \lambda_{i} e_{i}\right\| \leq\left\|\sum \lambda_{i} f_{i}\right\| \leq b\left\|\sum \lambda_{i} e_{i}\right\|$, in which case they are said to be $C$-equivalent. For basic sequences this is equivalent to saying that for any choice of reals $\left(\lambda_{i}\right)_{i \in \mathbb{N}}, \sum \lambda_{i} e_{i}$ converges if and only if $\sum \lambda_{i} f_{i}$ converges. We shall write $\left(e_{i}\right) \approx\left(f_{i}\right)$ to mean that $\left(e_{i}\right)$ and $\left(f_{i}\right)$ are equivalent.

Given some vector $x \in \operatorname{span}\left(e_{i}\right)$ let its support, $\operatorname{supp}(x)$, be the set of indices $i$ with $e_{i}^{*}(x) \neq 0$. For $k \in \mathbb{N}$ and $x, y \in \operatorname{span}\left(e_{i}\right)$ we write $k<x$ if $k<\min \operatorname{supp}(x)$, and $x<y$ if $\max \operatorname{supp}(x)<\min \operatorname{supp}(y)$. A block basis, $\left(x_{i}\right)$, over a basis $\left(e_{i}\right)$ is a finite or infinite sequence of vectors in $\operatorname{span}\left(e_{i}\right)$ with $x_{0}<x_{1}<x_{2}<\cdots$. This sequence will also be basic and in fact unconditional in case $\left(e_{i}\right)$ is so.

A space $\mathfrak{Y}$ is isomorphic to $\mathfrak{Z}$ if there is a bijective continuous linear map $T$ from $\mathfrak{Y}$ onto $\mathfrak{Z}$ such that $T^{-1}$ is also continuous. They are $C$-isomorphic if this happens for some $T$ such that $\|T\|\left\|T^{-1}\right\| \leq C$. The Banach-Mazur distance between $\mathfrak{Y}$ and $\mathfrak{Z}$, denoted by $d_{\mathrm{BM}}(\mathfrak{Y}, \mathfrak{Z})$ is defined as the infimum of the $C$ 's such that $\mathfrak{Y}$ and $\mathfrak{Z}$ are $C$-isomorphic. We shall write $\mathfrak{Y} \cong \mathfrak{Z}$ to mean that $\mathfrak{Y}$ and $\mathfrak{Z}$ are isomorphic, and $\mathfrak{Y} \cong_{K} \mathfrak{Z}$ to mean that they are $K$-isomorphic. 


\section{Models of separable Banach spaces and their basic sequences.}

To study separable Banach spaces by topological means we need some way to make a space out of them. So we turn to descriptive set theory for the basic tools. Let $\mathfrak{X}$ be a Polish space and let $F(\mathfrak{X})$ denote the set of closed subsets of $\mathfrak{X}$. We endow $F(\mathfrak{X})$ with the following $\sigma$-algebra that renders it a standard Borel space. The generators are the following sets, where $U$ varies over the open subsets of $\mathfrak{X}$ :

$$
\{F \in F(\mathfrak{X}) \mid F \cap U \neq \emptyset\} .
$$

The resulting measurable space is called the Effros Borel space of $F(\mathfrak{X})$ (see Section 12.E in [7]) .

A theorem due to Kuratowski and Ryll-Nardzewski ((12.13) in [7]) states that there is a sequence of Borel functions $d_{n}: F(\mathfrak{X}) \rightarrow \mathfrak{X}$ such that for nonempty $F \in F(\mathfrak{X})$ the set $\left\{d_{n}(F)\right\}$ is dense in $F$. Supposing now that $\mathfrak{X}$ is a separable Banach space, we can, in a Borel manner, express the fact that $F \in F(\mathfrak{X})$ is a linear subspace of $\mathfrak{X}$ :

$$
0 \in F \wedge \forall n, m \forall p, q \in \mathbb{Q}\left(p d_{n}(F)+q d_{m}(F) \in F\right) .
$$

We use here the fact that the relations $F_{0} \subset F_{1}$ and $x \in F$ are Borel in $F(\mathfrak{X})^{2}$ and $\mathfrak{X} \times F(\mathfrak{X})$ respectively. But then it is possible to construct a standard Borel space consisting of all separable Banach spaces. Simply take any isometrically universal separable Banach space (for example, $C\left(2^{\mathbb{N}}\right)$ ) and let $\mathfrak{A}$ be the set of closed linear subspaces of it. Call $\mathfrak{A}$ the Borel space of separable Banach spaces.

There is now a space in which we can express the relations of (linear) isomorphism, isometry, etc., and we will see that their descriptive complexities are as they should be, e.g., analytic for isomorphism.

If we restrict ourselves to certain types of subspaces, the situation is far less involved. Spaces spanned by subsequences of a given basis can be identified with $2^{\mathbb{N}}$. Also, following Gowers in [3], [4], it is natural to see the set $\mathfrak{b} \mathfrak{b}$ of normalised block sequences of a given basis as a closed subset of $\mathfrak{X}^{\mathbb{N}}$ equipped with the product of the norm topology. As expected, $\approx$ is Borel in $\mathfrak{b b}^{2}$ and the associated canonical injections from $2^{\mathbb{N}}$ and $\mathfrak{b} \mathfrak{b}$ into $\mathfrak{A}$ are Borel.

Going back to the notion of isomorphism, we see that it is indeed analytic: For $\mathfrak{X}, \mathfrak{Y} \in \mathfrak{A}$ we have $\mathfrak{X} \cong \mathfrak{Y}$ iff

$$
\begin{gathered}
\exists \boldsymbol{x} \exists \boldsymbol{y}\left(\boldsymbol{x} \approx \boldsymbol{y} \wedge \forall n x_{n} \in \mathfrak{X} \wedge \forall n y_{n} \in \mathfrak{Y} \wedge \forall n\left(\mathcal{U}_{n} \cap \mathfrak{X} \neq \emptyset \rightarrow \exists m x_{m} \in \mathcal{U}_{n}\right)\right. \\
\left.\wedge \forall n\left(\mathcal{U}_{n} \cap \mathfrak{Y} \neq \emptyset \rightarrow \exists m y_{m} \in \mathcal{U}_{n}\right)\right) .
\end{gathered}
$$

Here $\left\{\mathcal{U}_{n}\right\}_{n \in \mathbb{N}}$ is a basis for the topology on $C\left(2^{\mathbb{N}}\right)$.

Our first result concerns equivalence of subsequences (respectively block bases) of a given basis. 
3. Counting the number of non-equivalent block bases. Until now we have only been looking at Cantor's concept of cardinality, but there is also a newer and finer one stemming from descriptive set theory. It allows us to distinguish between different levels of $2^{\mathbb{N}}$ according to their complexity.

Definition 1. Let $E \subset \mathfrak{X}^{2}$ and $F \subset \mathfrak{Y}^{2}$ be equivalence relations on standard Borel spaces $\mathfrak{X}$ and $\mathfrak{Y}$. We say that $E$ is Borel reducible to $F$ $\left(E \leq_{B} F\right)$ if there is a Borel function $\phi: \mathfrak{X} \rightarrow \mathfrak{Y}$ such that $\forall x, y \in \mathfrak{X} x E y \leftrightarrow$ $\phi(x) F \phi(y)$. $E$ is Borel bireducible to $F\left(E \sim_{B} F\right)$ if both $E \leq_{B} F$ and $F \leq_{B} E$.

The definition is of interest only if $\mathfrak{X}$ and $\mathfrak{Y}$ are uncountable and $E$ and $F$ have uncountably many classes. Furthermore, it is usually supposed that the equivalence relations are of some bounded complexity, e.g., Borel or analytic.

Given some normalised basic sequence $\left(e_{i}\right)_{i \in \mathbb{N}}$ in a separable Banach space $\mathfrak{X}$, we can look at the set of its subsequences as a subspace of $2^{\mathbb{N}}$ : just take away the countable set FIN consisting of the finite subsets of $\mathbb{N}$ and identify a subsequence with its set of indices. $2^{\mathbb{N}} \backslash$ FIN is still a Polish space (though now non-compact) under the usual topology. Note that the relation of equivalence between subsequences of $\left(e_{i}\right)_{i \in \mathbb{N}}$ induces a Borel equivalence relation on $2^{\mathbb{N}} \backslash$ FIN.

An important measure of complexity is the following equivalence relation $E_{0}$, which is the minimum (up to $\sim_{B}$ ) Borel equivalence relation $\leq_{B}$-above the equality relation on $\mathbb{R}$ and is defined on $2^{\mathbb{N}}$ as follows:

$$
\alpha E_{0} \beta: \equiv \exists n \in \mathbb{N} \forall m \geq n \alpha_{m}=\beta_{m} .
$$

In particular, if $E_{0}$ Borel reduces to some equivalence relation $E$, then $E$ has continuum many classes.

Proposition 2. Let $\mathfrak{X}$ be a Banach space with a normalised basis $\left(e_{i}\right)_{i \in \mathbb{N}}$.

- Either $\left(e_{i}\right)_{i \in \mathbb{N}}$ is perfectly homogeneous, i.e., equivalent to all of its normalised block basic sequences (and therefore equivalent to the standard unit basis in some $\ell_{p}, 1 \leq p<\infty$, or $c_{0}$ ), or $E_{0}$ is Borel reducible to equivalence of its normalised block basic sequences.

- Either $\left(e_{i}\right)_{i \in \mathbb{N}}$ is subsymmetric, i.e., equivalent to all of its subsequences, or $E_{0}$ is Borel reducible to equivalence of its subsequences.

Proof. We show only the first part as the proof of the second is essentially the same.

Assume that $\mathfrak{X}$ has at least two non-equivalent normalised block sequences, $\left(x_{i}\right)$ and $\left(y_{i}\right)$. Then $\left(x_{i}\right)$ and $\left(y_{i}\right)$ are not 2-equivalent, so there exists $I_{1}$, an interval of integers, such that $\left(x_{i}\right)_{i \in I_{1}}$ and $\left(y_{i}\right)_{i \in I_{1}}$ are not 2equivalent. Also for any $k,\left(x_{i}\right)_{i>k}$ and $\left(y_{i}\right)_{i>k}$ are not equivalent, so there 
exists $k<I_{2} \subset \mathbb{N}$ such that $\left(x_{i}\right)_{i \in I_{2}}$ and $\left(y_{i}\right)_{i \in I_{2}}$ are not 4-equivalent. Without loss of generality, we may assume that $\operatorname{supp}\left(x_{i}, i \in I_{2}\right) \cup \operatorname{supp}\left(y_{i}, i \in I_{2}\right)$ $>\operatorname{supp}\left(x_{i}, i \in I_{1}\right) \cup \operatorname{supp}\left(y_{i}, i \in I_{1}\right)$. Repeating this, we get a sequence of successive intervals $I_{k}$ such that $\left(x_{i}\right)_{i \in I_{k}}$ and $\left(y_{i}\right)_{i \in I_{k}}$ are not $2^{k}$-equivalent.

For any $\alpha \in 2^{\mathbb{N}}$, let $f(\alpha)$ be the block basis $\left(f_{i}\right)$, indexed on $i \in \bigcup_{j \in \mathbb{N}} I_{j}$, defined by $f_{i}=x_{i}$ if $i \in I_{j}$ with $\alpha(j)=0$, and $f_{i}=y_{i}$ otherwise; clearly, $f(\alpha)$ is equivalent to $f(\beta)$ iff $\alpha E_{0} \beta$ and the map is Borel (even continuous).

Let us mention that in the case where $E_{0}$ Borel reduces to an analytic equivalence relation this latter can have no analytic transversal. That is, there can be no analytic set intersecting every equivalence class in exactly one point. So in particular, the above result says that if there is more than one class, then the relation is rather complicated, and cannot in fact be classified in a Borel manner by real numbers. Also, we refer to the forthcoming [2] for discussion about isomorphism on $\mathfrak{b} \mathfrak{b}$.

3.1. Classical Ramsey type results. We first mention that classical Ramsey type results can be proved in the case where there are countably many classes of isomorphism.

Lemma 3. For any $n$ there exists a constant $c(n)=1+n\left(2^{n+1}+1\right)^{2}$ such that for any Banach space $\mathfrak{X}$, all $n$-codimensional subspaces of $\mathfrak{X}$ are $c(n)$-isomorphic.

Proof. We are not interested in finding the best constant. We prove that $d_{\mathrm{BM}}\left(\mathfrak{X}, \mathfrak{Z} \oplus_{1} \ell_{2}^{n}\right) \leq \sqrt{n}\left(2^{n+1}+1\right)$ for any Banach space $\mathfrak{X}$ and any $n$-codimensional subspace $\mathfrak{Z}$ of $\mathfrak{X}$. The result follows: for any two $n$-codimensional subspaces $\mathfrak{H}$ and $\mathfrak{H}^{\prime}$ of a Banach space, there exists $W n$-codimensional in $\mathfrak{H}$ and $\mathfrak{H}^{\prime}$, so $\mathfrak{H}\left(\right.$ resp. $\left.\mathfrak{H}^{\prime}\right)$ is $\sqrt{n}\left(2^{n+1}+1\right)+\varepsilon$-isomorphic to $W \oplus_{1} \ell_{2}^{n}$ for all $\varepsilon>0$, and $d_{\mathrm{BM}}\left(\mathfrak{H}, \mathfrak{H}^{\prime}\right) \leq n\left(2^{n+1}+1\right)^{2}$.

So let $\mathfrak{X}$ be a Banach space and $\mathfrak{Z}$ be $n$-codimensional in $\mathfrak{X}$. By induction, there exists a projection $P$ on $\mathfrak{Z}$ of norm smaller than $2^{n}+\varepsilon$. Let $F=$ $(\operatorname{Id}-P)(\mathfrak{X})$. Then using $P$ one shows that $\mathfrak{X}=\mathfrak{Z} \oplus F$ is $2^{n+1}+1+2 \varepsilon$ isomorphic to $\mathfrak{Z} \oplus_{1} F$. By classical results, $F$ is $\sqrt{n}$-isomorphic to $\ell_{2}^{n}$, and it follows that $\mathfrak{X}$ is $\sqrt{n}\left(2^{n+1}+1+2 \varepsilon\right)$-isomorphic to $\mathfrak{Z} \oplus_{1} \ell_{2}^{n}$.

To spark confusion we will identify several different objects; namely the space $\left[e_{i}\right]_{i \in A}$ for some subset $A \subset \mathbb{N}$ with the characteristic function $\chi_{A}$ seen as a point in the Cantor space $2^{\mathbb{N}}$, which we again simply identify with the subset $A$ of $\mathbb{N}$. The subspaces spanned by subsequences are therefore equipped with the Polish topology inherited from $2^{\mathbb{N}}$. We denote by $A \cong B$ the fact that the corresponding Banach spaces are linearly isomorphic. If we see this relation as an equivalence relation between the points in the Effros Borel space of closed linear subspaces of $C\left(2^{\mathbb{N}}\right)$, it is analytic, and furthermore the function associating to $A \subset \mathbb{N}$ the space (or point) in the 
Effros Borel space is Borel. So we have an induced analytic equivalence relation, denoted by $\cong$, on $2^{\mathbb{N}}$. For $K \geq 1$ we write $A \cong_{K} B$ to denote that the corresponding spaces are $K$-isomorphic, so that $\cong$ is the union of the $\cong_{K}, K \in \mathbb{N}$. Recall that for a finite binary sequence $u=\left\langle u_{0}, \ldots, u_{k-1}\right\rangle, N_{u}$ denotes the basic open set of all $A \in 2^{\mathbb{N}}$ such that $i \in A$ iff $u_{i}=1$ for all $i<k$.

Proposition 4. Let $\mathfrak{X}$ be a Banach space with a basis $\left(e_{i}\right)_{i \in \mathbb{N}}$. If $\mathfrak{X}$ has countably many classes of isomorphism generated by subsequences of the basis then there exist $K$ and $A \subset \mathbb{N}$ such that $\left[e_{i}\right]_{i \in B} \cong_{K}\left[e_{i}\right]_{i \in A}$ for all $B \subset A$.

Proof. In this proof, we think of sets $A$ as increasing sequences of integers. For each $n$, let $E_{n} \subset \mathbb{N}$ be a representative in the $n$th $\cong$ class. Say that $A$ belongs to $\mathcal{A}_{m, n}$ if $A \cong_{m} E_{n}$; fix furthermore a bijection $k \mapsto\left(m_{k}, n_{k}\right)$ between $\mathbb{N}$ and $\mathbb{N}^{2}$.

Assume a Banach space $\mathfrak{X}$ contradicts the proposition and apply the infinite Ramsey theorem. No subset of $\mathbb{N}$ has all its subsets in $\mathcal{A}_{m_{0}, n_{0}}$, so there exists $A_{0} \subset \mathbb{N}$ such that no subset of $A_{0}$ is in $\mathcal{A}_{m_{0}, n_{0}}$. Let $k_{0}$ be an integer in $A_{0}$. No subset $A$ of $A_{0}$ after $k_{0}$ is such that $\left\{k_{0}\right\} \cup A$ has all its subsets in $\mathcal{A}_{m_{1}, n_{1}}$ : otherwise, any subset $\left\{b_{0}<b_{1}<\cdots\right\}$ of $A$ spans a space which is $c(1)$-isomorphic to the span of $\left\{k_{0}<b_{1}<\cdots\right\}$, so is $c(1) m_{0^{-}}$ isomorphic to $E_{n_{0}}$, a contradiction. So some subset $A_{1}$ of $A_{0}$ after $k_{0}$ is such that $\left\{k_{0}\right\} \cup A$ belongs to $\mathcal{A}_{m_{1}, n_{1}}$ for no $A \subset A_{1}$. Choose $k_{1}$ in $A_{1}$. Repeating this, define a decreasing sequence $\left\{A_{n}\right\}$ and an increasing sequence $\left\{k_{n}\right\}$ with $k_{i}$ in $A_{i}$ to get a set $K=\left\{k_{p}, p \in \mathbb{N}\right\}$ belonging to no $\mathcal{A}_{m, n}$.

The example of $\ell_{1} \oplus \ell_{2}$ proves that the isomorphism class in this proposition need not contain $\mathfrak{X}$, nor contain "most" subspaces of $\mathfrak{X}$; in fact, we shall see that the class of $\ell_{1}$ (respectively $\ell_{2}$ ) is meagre in the standard topology associated with the space.

\section{Banach spaces with unconditional basis isomorphic to their} squares. Let $\mathfrak{X}$ be a Banach space with a basis $\left(e_{i}\right)_{i \in \mathbb{N}}$. We will study the linear-isomorphism classes of spaces spanned by subsequences (finite and infinite) of this basis by using Baire category. As the finite subsets of $\mathbb{N}$ are fully characterised up to $\cong$ by their cardinality we will often forget about them. Also other relations on $2^{\mathbb{N}}$ will be useful. First note that the Cantor space is an abelian Polish group under the action of symmetric difference $\triangle$, with the identity element being $\emptyset$ and with each element being its own inverse. Therefore not only $\cong$ but also the relations

$$
A \sim_{1} B: \equiv A \cong \complement B, \quad A \sim_{2} B: \equiv \complement A \cong \complement B
$$

are analytic in $2^{\mathbb{N}}$. 
Theorem 5 (Kuratowski-Mycielski, (19.1) in [7]). Let $\mathfrak{X}$ be a perfect Polish space, and $R$ be a relation on $\mathfrak{X}$ meagre in $\mathfrak{X}^{2}$. Then there exists a homeomorphic copy $\mathcal{C}$ of the Cantor space such that for all $x, y \in \mathcal{C}$ with $x \neq y$ we have $\neg x R y$.

Suppose now that $\cong, \sim_{1}$ and $\sim_{2}$ are all meagre in the product $2^{\mathbb{N}} \times 2^{\mathbb{N}}$. Then so is their union and the theorem above gives us a Cantor set $\mathcal{C}$ avoiding the three relations. Now by taking away the countable set (possibly finite or empty) of finite sets in $\mathcal{C}$ and going to a further subset, we can suppose that $\mathcal{C} \cap \mathrm{FIN}=\emptyset$. This means in particular that there is a continuum of subsequences spanning non-isomorphic spaces.

Following Kalton we say that the basis $\left(e_{i}\right)_{i \in \mathbb{N}}$ is countably primary if there is a countable list $E_{0}, E_{1}, E_{2}, \ldots$ of Banach spaces such that if $A \subset \mathbb{N}$ then for some $n$ either $\left[e_{i}\right]_{i \in A} \cong E_{n}$ or $\left[e_{i}\right]_{i \in \mathbb{C} A} \cong E_{n}$. It is now easy to see that our space cannot be countably primary, for take two subsets of $\mathbb{N}$, $A \neq B, A, B \in \mathcal{C}$, associated to the same $E_{n}$, i.e., either $A \cong E_{n} \cong B$, $A \cong E_{n} \cong \complement B, \complement A \cong E_{n} \cong B$ or $\complement A \cong E_{n} \cong \complement B$. It should be evident for the reader taking the pains of unraveling our definitions that we get a minor contradiction here.

Going in the other direction we wish to see what we get from the fact that some of the three relations are non-meagre in the product. First we note that a Fubini type theorem is also true in Baire category (here $\exists^{*} x P(x)$ denotes the existence of a non-meagre set of $x$ such that $P(x))$ :

Theorem 6 (Kuratowski-Ulam, (8.41) in [7]). Let $\mathfrak{Y}$ be a Polish space and $\mathcal{D}$ a subset of the square having the Baire property. Then $\mathcal{D}$ is nonmeagre iff $\exists^{*} x \exists^{*} y(x, y) \in \mathcal{D}$ iff $\exists^{*} y \exists^{*} x(x, y) \in \mathcal{D}$.

Applying this to the above relations we get the following:

$$
\begin{aligned}
\cong \text { non-meagre in } 2^{\mathbb{N}} \times 2^{\mathbb{N}} & \Rightarrow \exists A \in 2^{\mathbb{N}} \exists^{*} B \in 2^{\mathbb{N}} A \cong B, \\
\sim_{1} \text { non-meagre in } 2^{\mathbb{N}} \times 2^{\mathbb{N}} & \Rightarrow \exists B \in 2^{\mathbb{N}} \exists^{*} A \in 2^{\mathbb{N}} A \cong \complement B \\
& \Rightarrow \exists C \in 2^{\mathbb{N}} \exists^{*} A \in 2^{\mathbb{N}} A \cong C, \\
\sim_{2} \text { non-meagre in } 2^{\mathbb{N}} \times 2^{\mathbb{N}} & \Rightarrow \exists A \in 2^{\mathbb{N}} \exists^{*} B \in 2^{\mathbb{N}} \complement A \cong \complement B \\
& \Rightarrow \exists C \in 2^{\mathbb{N}} \exists^{*} D \in 2^{\mathbb{N}} C \cong D .
\end{aligned}
$$

So each of the three cases gives us a non-meagre isomorphism class $\mathcal{A}$ in $2^{\mathbb{N}}$. Fix $B$ in $\mathcal{A}$ and for all $M \in \mathbb{N}$ denote by $\mathcal{A}_{M}$ the set $\left\{A \mid A \cong_{M} B\right\}$. Then for some $M$ large enough, $\mathcal{A}_{M}$ is non-meagre as well, and we will show that $\mathcal{A}_{K}$ for some $K \geq M$ (and therefore $\mathcal{A}$ ) is actually residual in the Cantor space.

Note that being a section of an analytic set, $\mathcal{A}_{M}$ is itself analytic and has therefore the property of Baire. So as it is non-meagre it must be residual in an open set $\mathcal{U} \subset 2^{\mathbb{N}}$, and by going to a smaller open set we can suppose 
that $\mathcal{U}$ is of the form $N_{s}$ for some finite binary sequence $s$. There are two features of $s$ interesting for us, its length and its cardinality. By the length, $|s|$, we mean its length as a sequence, and by the cardinality, $\bar{s}$, is meant the number of 1 's appearing in the sequence. We prove that $\mathcal{A}_{K}$ is residual for $K=M c(2|s|)$.

Otherwise, let $t \in 2^{<\mathbb{N}}$ be such that $\mathcal{A}_{K}$ is meagre in $N_{t}$. Without loss of generality, assume $|t| \geq|s|$ and write $t=u \frown v$ with $|u|=|s|$. Then let $t^{\prime}=t \frown s=u \frown v^{\frown} s$ and $s^{\prime}=s \frown v \frown u$. So $\left|t^{\prime}\right|=\left|s^{\prime}\right|, \overline{t^{\prime}}=\overline{s^{\prime}}$, and $\mathcal{A}_{M}$ is residual in $N_{s^{\prime}}$ while $\mathcal{A}_{K}$ is meagre in $N_{t^{\prime}}$.

We now have a natural homeomorphism $\phi$ between the clopen sets $N_{s^{\prime}}$ and $N_{t^{\prime}}$ : simply for an $A \in N_{s^{\prime}}$ change the beginning from $s^{\prime}$ to $t^{\prime}$, i.e. $\phi\left(s^{\prime} \frown \alpha\right)=t^{\prime} \propto \alpha$, where $\chi_{A}=s^{\prime} \propto \alpha$. By construction, $s^{\prime} \propto \alpha$ and $t^{\prime \frown} \alpha$ code subspaces of the same codimension $2|s|-\bar{s}-\bar{u} \leq 2|s|$ of the space coded by $\left(1^{|s|}\right) \frown v \frown\left(1^{|s|}\right) \frown \alpha$ (here $1^{n}$ denotes the length $n$ sequence of 1 's). By Lemma 3 , it follows that $\phi(A) \cong{ }_{c(2|s|)} A$. As $\mathcal{A}_{M}$ is residual in $N_{s^{\prime}}$, it follows that $\mathcal{A}_{c(2|s|) M} \supset \phi\left(\mathcal{A}_{M}\right)$ is residual in $N_{t^{\prime}}$, which is a contradiction.

We now need a standard compactness result from descriptive set theory:

LEMMA 7. If $\mathcal{G}$ is a residual subset of $2^{\mathbb{N}}$, then there exists a partition $A_{0}, A_{1}, A_{2}, \ldots$ of $\mathbb{N}$ and subsets $B_{i} \subset A_{i}, i \in \mathbb{N}$, such that for any set $E \subset \mathbb{N}$, if $E \cap A_{i}=B_{i}$ for some $i \in \mathbb{N}$ then $E \in \mathcal{G}$.

Proof. For $\mathcal{D}$ a dense open set in $2^{\mathbb{N}}$ and $n \in \mathbb{N}$ there is $s \in 2^{<\mathbb{N}}$ such that for any $t \in 2^{n}$ we have $N_{t}{ }_{s} \subset \mathcal{D}$.

This is because we can enumerate $2^{n}$ as $\left\{t_{1}, t_{2}, \ldots, t_{2^{n}}\right\}$ and hence, as $\mathcal{D}$ is dense open, there is some $s_{1} \in 2^{<\mathbb{N}}$ with $N_{t_{1} s_{1}} \subset \mathcal{D}$. Now find $s_{2} \in 2^{<\mathbb{N}}$ with $N_{t_{2}} s_{1} s_{2} \subset \mathcal{D}$. Again find $s_{3} \in 2^{<\mathbb{N}}$ with $N_{t_{3}} s_{1} s_{2} s_{3} \subset \mathcal{D}$, etc. Finally, putting $s=s_{1} s_{2} \ldots \frown s_{2^{n}}$ yields our result.

Suppose that $\mathcal{G} \supset \bigcap_{i \in \mathbb{N}} \mathcal{D}_{i}$ for dense open sets $\mathcal{D}_{i}$.

- Let $n(0,0)=0$.

- Take $s(0,0)$ such that $N_{s(0,0)} \subset \mathcal{D}_{0}$, put $n(1,0)=n(0,0)+|s(0,0)|$.

- Take $s(1,0)$ such that $N_{t}-s(1,0) \subset \mathcal{D}_{0}, \forall t \in 2^{n(1,0)}$, put $n(0,1)=n(1,0)+|s(1,0)|$.

- Take $s(0,1)$ such that $N_{t}-s(0,1) \subset \mathcal{D}_{1}, \forall t \in 2^{n(0,1)}$, put $n(2,0)=n(0,1)+|s(0,1)|$.

- Take $s(2,0)$ such that $N_{t}-s(2,0) \subset \mathcal{D}_{0}, \forall t \in 2^{n(2,0)}$, put $n(1,1)=n(2,0)+|s(2,0)|$.

- Take $s(1,1)$ such that $N_{t}-s(1,1) \subset \mathcal{D}_{1}, \forall t \in 2^{n(1,1)}$, put $n(0,2)=n(1,1)+|s(1,1)|$.

- Take $s(0,2)$ such that $N_{t}-s(0,2) \subset \mathcal{D}_{2}, \forall t \in 2^{n(0,2)}$, put $n(3,0)=n(0,2)+|s(0,2)|$, 
etc. Finally, set

$$
\begin{aligned}
& A_{i}:=\bigcup_{k \in \mathbb{N}}[n(i, k), n(i, k)+|s(i, k)|[ \\
& B_{i}:=\bigcup_{k \in \mathbb{N}}\{n(i, k)+m \mid(s(i, k))(m)=1\}
\end{aligned}
$$

Letting $A_{0}^{\prime}:=A_{0}, A_{1}^{\prime}:=\bigcup_{i=1}^{\infty} A_{i}, B_{0}^{\prime}:=B_{0}, B_{1}^{\prime}:=\bigcup_{i=1}^{\infty} B_{i}$ we get: If $\mathcal{G}$ is a residual set in $2^{\mathbb{N}}$, then there is a partition $\mathbb{N}=A_{0}^{\prime} \cup A_{1}^{\prime}, A_{0}^{\prime} \cap A_{1}^{\prime}=\emptyset$ and sets $B_{i}^{\prime} \subset A_{i}^{\prime}, i \in 0,1$, such that for any $D \subset \mathbb{N}$, if either $D \cap A_{0}^{\prime}=B_{0}^{\prime}$ or $D \cap A_{1}^{\prime}=B_{1}^{\prime}$ we have $D \in \mathcal{G}$.

We shall now assume the basis is unconditional. Apply the lemma to $\mathcal{A}$ and notice that $B_{0}, B_{1}$ and $B_{0} \cup B_{1}$ are in $\mathcal{A}$. Now as $\left(e_{i}\right)_{i \in \mathbb{N}}$ is unconditional, for any pair of disjoint sets $C, D \subset \mathbb{N}$ we have $\left[e_{i}\right]_{i \in C} \oplus\left[e_{i}\right]_{i \in D} \cong\left[e_{i}\right]_{i \in C \cup D}$. Moreover, since $\mathcal{A}$ is residual and $\complement$ is a homeomorphism of $2^{\mathbb{N}}$ there is some $C \subset \mathbb{N}$ with $C, \complement C \in \mathcal{A}$. So again abusing notation we calculate

$$
\mathbb{N} \cong C \cup \complement C \cong C \oplus \complement C \cong B_{0} \oplus B_{0} \cong B_{0} \oplus B_{1} \cong B_{0} \cup B_{1} \cong B_{0} .
$$

Hence $\mathbb{N}$ belongs to the residual class $\mathcal{A}$ and $\left[e_{i}\right]_{i \in \mathbb{N}}$ is isomorphic to $\left[e_{i}\right]_{i \in B_{0}}$, which is isomorphic to its square.

Without loss of generality assume $\mathbb{N}$ belongs to $\mathcal{A}_{K}$ and let $c$ be the unconditionality constant of the basis. We denote by $\oplus_{1}$ the $\ell_{1}$-sum of Banach spaces.

Again for any subset $D \subset \mathbb{N}$, since $A_{0}, A_{1}$ partition $\mathbb{N}$, we have

$$
\begin{aligned}
\mathbb{N} \oplus_{1} D & \cong_{K}\left(B_{0} \cup B_{1}\right) \oplus_{1}\left(\left(D \cap A_{1}\right) \cup\left(D \cap A_{0}\right)\right) \\
& \cong_{2 C} B_{0} \oplus_{1} B_{1} \oplus_{1}\left(D \cap A_{1}\right) \oplus_{1}\left(D \cap A_{0}\right) \\
& \cong_{2 C}\left(B_{0} \cup\left(D \cap A_{1}\right)\right) \oplus_{1}\left(B_{1} \cup\left(D \cap A_{0}\right)\right) \cong_{K} B_{0} \oplus_{1} B_{1} \cong_{K} \mathbb{N} .
\end{aligned}
$$

So spaces of the form $\left[e_{i}\right]_{i \in \mathbb{N}} \oplus_{1}\left[e_{i}\right]_{i \in D}$ for any $D \subset \mathbb{N}$ are $4 C^{2} K^{3}$-isomorphic to $\left[e_{i}\right]_{i \in \mathbb{N}}$; and in particular $\left[e_{i}\right]_{i \in \mathbb{N}}$ is isomorphic to its hyperplanes.

Using also the complete version of the above lemma, we find

$$
\bigcup_{i \in \mathbb{N}} B_{i}, B_{0}, B_{1}, B_{2}, \ldots \in \mathcal{A} .
$$

But $\left[e_{i}\right]_{i \in B_{0}},\left[e_{i}\right]_{i \in B_{1}},\left[e_{i}\right]_{i \in B_{2}}, \ldots$ gives (due to the unconditionality of the basis) an unconditional Schauder decomposition of $\left[e_{i}\right]_{i \in \cup_{j \in \mathbb{N}} B_{j}}$ and this latter is again isomorphic to $\left[e_{i}\right]_{i \in \mathbb{N}}$.

Summing up we have arrived at the following:

THEOREM 8. Let $\mathfrak{X}$ be a Banach space with an unconditional basis $\left\{e_{i}\right\}_{i \in \mathbb{N}}$. Then either: 
(1) There exists a perfect set $\mathcal{P} \subset 2^{\mathbb{N}}$ of infinite subsets of $\mathbb{N}$ such that for any two distinct $A, B \in \mathcal{P}$ we have

$$
\left[e_{i}\right]_{i \in A} \nsucceq\left[e_{i}\right]_{i \in B}, \quad\left[e_{i}\right]_{i \in A} \nsucceq\left[e_{i}\right]_{i \in \mathbf{C} B}, \quad\left[e_{i}\right]_{i \in \mathbf{C} A} \nsucceq\left[e_{i}\right]_{i \in \mathbf{C} B},
$$

or

(2) For $A$ in a residual subset of $2^{\mathbb{N}},\left[e_{i}\right]_{i \in A}$ is isomorphic to $\mathfrak{X} ; \mathfrak{X}$ is isomorphic to its hyperplanes, to its square, uniformly isomorphic to $\mathfrak{X} \oplus$ $\left[e_{i}\right]_{i \in D}$ for any $D \subset \mathbb{N}$ and to a denumerable Schauder decomposition into uniformly isomorphic copies of itself, $\mathfrak{X} \cong\left(\sum_{k \in \mathbb{N}} \oplus\left(\left[e_{i}\right]_{i \in B_{k}}\right)\right)$.

As we noticed earlier, (1) in our dichotomy implies that the least cardinal $\kappa$ such that the basis is $\kappa$-primary (with the obvious definition) is $2^{\mathbb{N}}$, that is, the trivial one.

The theorem improves on an earlier result by Kalton which gives (2), without uniformity, in case the unconditional basis is countably primary. His proof, like the above, used very little from Banach space theory, but instead his setting was measure and probability theory [6].

As is easily seen from the proof, (1) can be strengthened considerably, and for our purposes, in fact one can get a perfect set avoiding any countable list of relations of the form

$$
\left[e_{i}\right]_{i \in A} \cong \phi(B),
$$

where $\phi$ is a Borel function from $2^{\mathbb{N}}$ to the Effros Borel space of closed linear subspaces of $C\left(2^{\mathbb{N}}\right)$, that is, the canonical space of separable Banach spaces. For example, one could use Borel functions $\psi$ from $2^{\mathbb{N}}$ to the space $\mathfrak{b} \mathfrak{b}$ of normalised block sequences of some given basis, and one would avoid

$$
\left[e_{i}\right]_{i \in A} \cong[\psi(B)] \text {. }
$$

E.g., we can force

$$
\left[e_{i}\right]_{i \in A} \nsucceq\left(\left[e_{i}\right]_{i \in B}\right)^{2}, \quad\left[e_{i}\right]_{i \in A} ¥\left(\left[e_{i}\right]_{i \in B}\right)^{3}, \quad\left[e_{i}\right]_{i \in A} \nsucceq\left(\left[e_{i}\right]_{i \in B}\right)^{4},
$$

and so on. $\mathrm{Or}$

$$
\left[e_{i}\right]_{i \in A} ¥ c_{0}\left(\left[e_{i}\right]_{i \in B}\right)
$$

or whatever construction from $B$ being reasonably explicit.

EXAMPLE 9. In a certain sense, the above result is optimal. For we might try to show that not only is some isomorphism class residual in $2^{\mathbb{N}}$, but that it is all of $2^{\mathbb{N}} \backslash$ FIN. However, this is easily seen to be false, for take the following basis for $\ell_{1} \oplus \ell_{2}$ :

$$
\begin{aligned}
\| a_{0} e_{0}+a_{1} e_{1}+\cdots & +a_{2 n+1} e_{2 n+1} \| \\
& :=\left|a_{0}\right|+\left|a_{2}\right|+\cdots+\left|a_{2 n}\right|+\sqrt{a_{1}^{2}+a_{3}^{2}+\cdots+a_{2 n+1}^{2}} .
\end{aligned}
$$

Then there are exactly three isomorphism classes: $\ell_{1}, \ell_{2}$, and $\ell_{1} \oplus \ell_{2}$, the first two being meagre in $2^{\mathbb{N}}$. Because if $A \subset \mathbb{N}, A$ infinite, contains infinitely 
many even and odd numbers then $\left[e_{i}\right]_{i \in A} \cong \ell_{1} \oplus \ell_{2}$, if it only contains finitely many even numbers then $\left[e_{i}\right]_{i \in A} \cong \ell_{2}$, and if only finitely many odd numbers then $\left[e_{i}\right]_{i \in A} \cong \ell_{1}$.

If we take the standard Haar basis for some $L_{p}([0,1]), 1<p<\infty$, then it is unconditional and in fact the only two spaces spanned by subsequences are $\ell_{p}$ and $L_{p}([0,1])$; so there are bases inducing exactly two isomorphism classes in $2^{\mathbb{N}} \backslash$ FIN (see [9, Thm. 2.d.10]).

EXAMPLE 10 (Tsirelson's space). We take a look at the standard unit vector basis for the Tsirelson space. It has the following properties (see [1]; here $\left.\mathbb{N}^{*}=\mathbb{N} \backslash\{0\}\right)$ :

- Two subsequences $\left(t_{k_{i}}\right)_{i \in \mathbb{N}^{*}}$ and $\left(t_{l_{i}}\right)_{i \in \mathbb{N}^{*}}$ are equivalent iff

$$
\left[t_{k_{i}}\right]_{i \in \mathbb{N}^{*}} \cong\left[t_{l_{i}}\right]_{i \in \mathbb{N}^{*}} .
$$

- Two subsequences $\left(t_{k_{i}}\right)_{i \in \mathbb{N}^{*}}$ and $\left(t_{l_{i}}\right)_{i \in \mathbb{N}^{*}}$ are equivalent iff

$$
\sup _{i \in \mathbb{N}^{*}}\left(\left\|I_{i}\right\|,\left\|J_{i}\right\|\right)<\infty
$$

where

$$
I_{i}:\left[t_{l_{n}} \mid k_{i-1}<l_{n} \leq k_{i}\right] \hookrightarrow \ell_{1}
$$

and

$$
J_{i}:\left[t_{k_{k_{n}}} \mid l_{i-1}<k_{n} \leq l_{i}\right] \hookrightarrow \ell_{1}
$$

are the formal identities $\left(k_{0}:=l_{0}:=0\right)$.

- $\left(t_{i}\right)_{i \in \mathbb{N}^{*}}$ and $\left(t_{k_{i}}\right)_{i \in \mathbb{N}^{*}}$ are equivalent iff the function $k: \mathbb{N}^{*} \rightarrow \mathbb{N}^{*}$ is majorised by a primitive recursive function.

We can view subsets $A \subset \mathbb{N}^{*}$ as strictly increasing functions $a: \mathbb{N}^{*} \rightarrow \mathbb{N}^{*}$ : simply let $a$ enumerate $A$ in the usual order. In the same way, strictly increasing functions can be seen as infinite subsets of $\mathbb{N}^{*}$. Now the relation of $b$ majorising $a$ is in fact closed in $\left(2^{\mathbb{N}} \backslash \mathrm{FIN}\right)^{2}$ and has closed, nowhere dense sections:

$$
\begin{aligned}
a \leq b \equiv \forall n a(n) \leq & b(n) \\
\equiv \forall n \forall m, k[ & {\left[\forall m_{1}<\cdots<m_{n}<m \exists i B\left(m_{i}\right)=0 \wedge\right.} \\
& \exists m_{1}<\cdots<m_{n}=m \forall i B\left(m_{i}\right)=1 \wedge \\
& \forall k_{1}<\cdots<k_{n}<k \exists i A\left(k_{i}\right)=0 \wedge \\
& \left.\left.\exists k_{1}<\cdots<k_{n}=k \forall i A\left(k_{i}\right)=1\right] \rightarrow k \leq m\right] .
\end{aligned}
$$

So it is closed and it is easily seen that no function $a$ can be such that it majorises all functions belonging to some basic open set $N_{s}$ (on the other hand, $n \mapsto n$ minorises all functions), whence the sections (in one of the coordinates) have empty interior. The set $\left\{A \subset \mathbb{N}^{*} \mid A \cong \mathbb{N}^{*}\right\}$ is the countable union of closed nowhere dense sets, hence meagre. 
Now take any $A \neq \mathbb{N}^{*}, A=\left\{a_{n}\right\}_{n \in \mathbb{N}^{*}}$. As it generates a sequence not equivalent to the full basis, there are disjoint non-empty intervals

$$
\left[r_{1}, s_{1}\left[,\left[r_{2}, s_{2}[, \ldots \subset \complement A\right.\right.\right.
$$

such that for the formal identities

$$
I_{i}:\left[t_{n} \mid n \in\left[r_{i}, s_{i}\right]\right] \hookrightarrow \ell_{1}
$$

we have $\left\|I_{i}\right\|>i$. So if $B \cong A$ then $\exists i \forall j \geq i\left[r_{j}, s_{j}\right] \not \subset B$, which is again easily seen to be the countable disjunction of closed, nowhere dense conditions.

Therefore every isomorphism class for the Tsirelson space is meagre in $2^{\mathbb{N}}$. However, using the functions $n \mapsto 2 n$ and $n \mapsto 2 n+1$, it is seen that the space is isomorphic to its square.

Though the notion of "complement" of a set is not absolute, i.e., it depends on the ambient space, $\cong$ is so. This is to say, the fact that $A \cong B$ does not depend on whether $A$ and $B$ are seen as subsets of $\mathbb{N}$ or of any other $C \subset \mathbb{N}$.

Denote by $2^{A}$ the closed set $\left\{\chi_{B} \in 2^{\mathbb{N}} \mid B \subset A\right\}$. If $A$ is infinite then this set is homeomorphic to $2^{\mathbb{N}}$ and we can use our preceding arguments.

So if for some $A$ the restriction of $\cong$ to $2^{A}$ is meagre in $2^{A}$ there are $2^{\mathbb{N}}$ isomorphism classes generated by subsequences of $A$, hence also of $\mathbb{N}$.

If not, there is some isomorphism class residual in $2^{A}$ and we deduce as before that $\left[e_{i}\right]_{i \in A}$ is isomorphic to its hyperplanes, to its square, to $\left[e_{i}\right]_{i \in A} \oplus$ $\left[e_{i}\right]_{i \in D}$ for any $D \subset A$, and to a denumerable Schauder decomposition into isomorphic copies of itself, $\left[e_{i}\right]_{i \in A} \cong\left(\sum_{k \in \mathbb{N}} \oplus\left(\left[e_{i}\right]_{i \in B_{k}}\right)\right)$.

There is a priori no control on the uniformity of the isomorphisms between each space $\left[e_{i}\right]_{i \in A}$ and its square. Notice also that the existence of an isomorphism $A \oplus D \cong A$ for $D$ an infinite subset of $A$ is straightforward from the assumption that $A \cong A \oplus A$ for all infinite $A \subset \mathbb{N}$. For then $A \oplus D \cong(A \backslash D) \oplus D \oplus D \cong(A \backslash D) \oplus D \cong A$, for all infinite $D \subset A$.

The diligent reader is invited to amuse himself by applying the above proof to the cases of Lipschitz homeomorphism, uniform homeomorphism and permutative equivalence of bases (all three are analytic equivalence relations).

THEOREM 11. Let $\mathfrak{X}$ be a Banach space with an unconditional basis $\left(e_{i}\right)_{i \in \mathbb{N}}$. Then either:

(1) There exists a perfect set $\mathcal{P} \subset 2^{\mathbb{N}}$ of infinite subsets of $\mathbb{N}$ such that for any two distinct $A, B \in \mathcal{P}$ we have

$$
\left[e_{i}\right]_{i \in A} \not\left[e_{i}\right]_{i \in B},
$$

or 
(2) For any infinite subset $A \subset \mathbb{N},\left[e_{i}\right]_{i \in A}$ is isomorphic to its hyperplanes, to its square, and to a denumerable Schauder decomposition into uniformly isomorphic copies of itself, $\left[e_{i}\right]_{i \in A} \cong\left(\sum_{k \in \mathbb{N}} \oplus\left(\left[e_{i}\right]_{i \in B_{k}}\right)\right)$.

Recall that a Banach space is complementably minimal if it embeds complementably in any of its subspaces. Using Pełczyński's decomposition method and the above result, one proves:

COROLlary 12. Let $\mathfrak{X}$ be a complementably minimal Banach space with an unconditional basis $\left(e_{i}\right)_{i \in \mathbb{N}}$. Then either:

(1) There exists a perfect set $\mathcal{P} \subset 2^{\mathbb{N}}$ of infinite subsets of $\mathbb{N}$ such that for any two distinct $A, B \in \mathcal{P}$ we have

$$
\left[e_{i}\right]_{i \in A} ¥\left[e_{i}\right]_{i \in B},
$$

or

(2) For any infinite subset $A \subset \mathbb{N},\left[e_{i}\right]_{i \in A} \cong \mathfrak{X}$.

4.1. The number of non-isomorphic subspaces of hereditarily indecomposable Banach spaces. Let now $\mathfrak{X}$ be a separable hereditarily indecomposable (H.I.) Banach space. This means that no (closed, infinite-dimensional) subspace of $\mathfrak{X}$ can be written as a direct sum of two closed infinite-dimensional subspaces. In this section "space" will always refer to closed infinitedimensional subspaces of $\mathfrak{X}$. It follows clearly from the H.I. property that $\mathfrak{X}$ contains no unconditional basic sequence; and in fact, by Gowers's dichotomy theorem, every Banach space contains either an H.I. subspace or a subspace with an unconditional basis. Moreover, Gowers and Maurey proved that an H.I. space is isomorphic to no proper subspace (and as the H.I. property is hereditary, this is also true of any subspace of $\mathfrak{X}$ ).

Now since we have the first property we cannot hope to use the above theorem to conclude something about the number of non-isomorphic subspaces of $\mathfrak{X}$, but it is still possible to use the fact that $\mathfrak{X}$ must contain some basic sequence $\left(e_{i}\right)_{i \in \mathbb{N}}$. Again we look at the subspaces spanned by subsequences as points in $2^{\mathbb{N}}$.

According to Dedekind every real is a set of rational numbers $r=$ $\{q \in \mathbb{Q} \mid q<r\}$, but following Cantor the set of rational numbers is the same as the set of natural numbers. So every real $r$ is a set $A_{r}$ of natural numbers such that $r<s \equiv A_{r} \subsetneq A_{s}$. Now again confusing Banach spaces with subsets of $\mathbb{N}$, reals become for us a subspace $\mathfrak{B}_{r}$ and the relation $r<s$ is simply strict inclusion $\mathfrak{B}_{r} \subsetneq \mathfrak{B}_{s}$. All of the identifying functions are evidently Borel. So we have $2^{\mathbb{N}}$ non-isomorphic subspaces of $\mathfrak{X}$.

This could also be seen by using the arguments from the preceding section. For suppose that some isomorphism class $\mathcal{A}$ was residual in $2^{\mathbb{N}}$. Then 
as $\{0\} \triangle(\cdot)$ is a homeomorphism of $2^{\mathbb{N}}$,

$$
\mathcal{A} \cap\{\{0\} \triangle A \mid A \in \mathcal{A}\} \neq \emptyset .
$$

So there is some $B \subset \mathbb{N}, 0 \notin B$ with $B,\{0\} \cup B \in \mathcal{A}$, i.e., some subspace of $\mathfrak{X}$ isomorphic to a hyperplane. But this cannot be the case in an H.I. space, so $\cong$ must be meagre in $2^{\mathbb{N}}$ and the Kuratowski-Mycielski result takes care of the rest.

Proposition 13. Any H.I. Banach space contains $2^{\mathbb{N}}$ pairwise non-isomorphic subspaces.

Corollary 14. Any Banach space contains $2^{\mathbb{N}}$ pairwise non-isomorphic subspaces or is saturated with subspaces isomorphic to their squares.

\section{References}

[1] P. G. Casazza and T. Shura, Tsirelson's Space, Lecture Notes in Math. 1363, Springer, 1989.

[2] V. Ferenczi and C. Rosendal, Ergodic Banach spaces, Adv. Math., to appear.

[3] W. T. Gowers, A new dichotomy for Banach spaces, Geom. Funct. Anal. 6 (1996), 1083-1093.

[4] -, An infinite Ramsey Theorem and some Banach-space dichotomies, Ann. of Math. 156 (2002), 797-833.

[5] W. T. Gowers and B. Maurey, The unconditional basic sequence problem, J. Amer. Math. Soc. 6 (1993), 851-874.

[6] N. Kalton, A remark on Banach spaces isomorphic to their squares, in: Contemp. Math. 232, Amer. Math. Soc., 1999, 211-217.

[7] A. Kechris, Classical Descriptive Set Theory, Springer, New York, 1995.

[8] R. Komorowski and N. Tomczak-Jaegermann, Banach spaces without local unconditional structure, Israel J. Math. 89 (1995), 205-226; Erratum, ibid. 105 (1998), 85-92.

[9] J. Lindenstrauss and L. Tzafriri, Classical Banach Spaces II, Springer, Berlin, 1979.

[10] N. Tomczak-Jaegermann, Banach spaces with many isomorphisms, Seminário Brasileiro de Análise 48, Petropolis, Rio de Janeiro, 1998, 189-210.

Equipe d'Analyse

Université Paris 6

Couloir 46-0, Boîte 186

4 , place Jussieu

75252 Paris Cedex 05, France

E-mail: ferenczi@ccr.jussieu.fr rosendal@ccr.jussieu.fr
Current address of C. Rosendal: Mathematics 253-37

California Institute of Technology Pasadena, CA 91125, U.S.A.

Received March 11, 2002

Revised version January 27, 2005 